\title{
Adding machines, endpoints, and inverse limit spaces
}

\author{
by
}

Lori Alvin and Karen Brucks (Milwaukee, WI)

\begin{abstract}
Let $f$ be a unimodal map in the logistic or symmetric tent family whose restriction to the omega limit set of the turning point is topologically conjugate to an adding machine. A combinatoric characterization is provided for endpoints of the inverse limit space $(I, f)$, where $I$ denotes the core of the map.
\end{abstract}

1. Introduction. The topological structure of inverse limit spaces with unimodal bonding maps has received great interest in recent years. Ingram's Conjecture, dating to the early 1990's, states that inverse limit spaces $(I, f)$ and $(I, g)$ are not topologically homeomorphic when $f$ and $g$ are distinct symmetric tent maps [8]. Kailhofer [10] first proved the conjecture in the case where the turning point is periodic. Stimac [13] then provided a proof when the turning point is of finite order, and later Raines and Stimac [12] proved the conjecture when the turning point is non-recurrent. Most recently, Barge, Bruin and Štimac [1] established Ingram's Conjecture.

An investigation of inverse limit spaces often involves endpoints, as they are a topological invariant. Barge and Martin [2] provide a topological characterization of endpoints for inverse limit spaces $(I, f)$ where $f$ is a continuous self-map of a closed interval. A characterization with both a combinatoric and analytic component is provided by Bruin [6] when $f$ is unimodal and the turning point is not periodic. The combinatoric component uses the kneading sequence of the bonding map $f$. In this paper we establish that the combinatoric component of Bruin's characterization suffices in the case where $f$ is unimodal and $\left.f\right|_{\omega(c)}$ is topologically conjugate to an adding machine.

Given a unimodal map $f$ with recurrent turning point $c$, set

$$
\mathcal{E}=\left\{x=\left(x_{0}, x_{1}, \ldots\right) \in(I, f) \mid x_{i} \in \omega(c) \text { for all } i \in \mathbb{N}\right\} .
$$

2010 Mathematics Subject Classification: Primary 54H20, 37B45; Secondary 37E05. Key words and phrases: adding machines, inverse limit spaces, endpoints. 
It is not difficult to show that the set of endpoints of $(I, f)$ is contained in $\mathcal{E}$. Here $I$ is understood to be the "core" of the map $f$. There are no endpoints in the case where $c$ is not recurrent with the exception of the symmetric tent or logistic map with kneading sequence $10^{\infty}$ [2]. Let $\mathcal{A}$ denote the collection of unimodal maps $f$ where $\left.f\right|_{\omega(c)}$ is topologically conjugate to an adding machine. In the case where $f \in \mathcal{A}$ is infinitely renormalizable, we use our characterization to establish that $\mathcal{E}$ is precisely the collection of endpoints of $(I, f)$. In addition, using our characterization, we prove there exist symmetric tent maps $f \in \mathcal{A}$ for which the collection of endpoints of $(I, f)$ is a proper subset of $\mathcal{E}$.

Given an infinitely renormalizable map $f$ in the logistic family with turning point $c$ it is known that $\left.f\right|_{\omega(c)}$ is topologically conjugate to an adding machine; see for example [11, Proposition III.4.5]. It was unknown whether adding machines could be embedded in the non-infinitely renormalizable case until 2006 when Block et al. 44 proved that adding machines can be embedded in symmetric tent maps. Indeed these embeddings occur for a dense set of parameters in the interval $[1,2]$. Adding machines embedded in non-infinitely renormalizable unimodal maps are called strange adding machines (SAM) [4].

In [4] an SAM scheme is defined and this SAM scheme is used to show the existence of adding machines embedded in maps from the symmetric tent family. It is precisely the symmetric tent maps obtained with an SAM scheme for which we prove that $\mathcal{E}$ contains non-endpoints of $(I, f)$. In contrast, as noted above, we prove that if $f$ is an infinitely renormalizable logistic map, then $\mathcal{E}$ is precisely the collection of endpoints of $(I, f)$. An alternative proof for the latter is provided in [9]; however the approach does not lend itself to investigating the SAM setting.

It is known that if $f:[0,1] \rightarrow[0,1]$ is unimodal with a closed invariant subset $X$ such that $\left.f\right|_{X}$ is topologically conjugate to an adding machine, then the turning point $c$ is in $X$ (for a short proof see [4]). Hence in searching for adding machines embedded in unimodal maps, it suffices to consider $\left.f\right|_{\omega(c)}$. A set $S \subset[0,1]$ is said to straddle $c$ if $S$ contains points lying on both sides of $c$ but does not contain $c$. An SAM scheme produces a set that straddles $c$ and it is precisely this set that we exploit to establish that $\mathcal{E}$ contains non-endpoints in the symmetric tent case.

Throughout this note it is assumed that all maps are unimodal, have no wandering intervals, and have no attracting periodic orbits. We note that if such a unimodal map $f$ is not renormalizable, then $f$ is topologically conjugate to a symmetric tent map. In the renormalizable case, $f$ may be taken to be in the logistic family [5]. 


\section{Background}

2.1. Unimodal maps. A continuous function $f:[0,1] \rightarrow[0,1]$ for which there exists a point $c \in(0,1)$ such that $\left.f\right|_{[0, c]}$ is strictly increasing and $\left.f\right|_{[c, 1]}$ is strictly decreasing is called unimodal. This point $c$ is called the turning point and we set $c_{i}=f^{i}(c)$ for all $i \in \mathbb{N}$. Examples of unimodal maps include symmetric tent and logistic maps. The symmetric tent map $T_{a}:[0,1] \rightarrow[0,1]$ with $a \in[0,2]$ is given by

$$
T_{a}(x)= \begin{cases}a x & \text { if } x \leq 1 / 2 \\ a(1-x) & \text { if } x \geq 1 / 2\end{cases}
$$

The logistic map $g_{a}:[0,1] \rightarrow[0,1]$ with $a \in[0,4]$ is defined by $g_{a}(x)=$ ax $(1-x)$.

Given a unimodal map $f$ and $x \in[0,1]$, the itinerary of $x$ under $f$ is given by $I(x)=I_{0} I_{1} I_{2} \cdots$ where $I_{j}=1$ if $f^{j}(x)>c, I_{j}=0$ if $f^{j}(x)<c$, and $I_{j}=*$ if $f^{j}(x)=c$. We make the convention that the itinerary stops after the first $*$ appears, and hence if $f^{n}(x) \neq c$ for all $n$, then $I(x)$ is infinite. The kneading sequence of a map $f$, denoted $\mathcal{K}(f)$, is the sequence $I(f(c))$. For ease of notation we set $\mathcal{K}(f)=e_{1} e_{2} e_{3} \cdots$, and therefore $e_{i}$ indicates the position of $c_{i}$ relative to $c$. For $x \in[0,1]$ we let $\omega(x, f)=\omega(x)=\{y \in[0,1] \mid$ there exists $n_{1}<n_{2}<\cdots$ with $\left.f^{n_{i}}(x) \rightarrow y\right\}$ denote the omega limit set of $x$ under $f$.

A unimodal map $f$ (with turning point $c$ ) is renormalizable provided there exists a restrictive interval $J \ni c$ and an $n \geq 2$ such that $f^{n}(J) \subset J$ and $\left.f^{n}\right|_{J}$ is again unimodal. If we may repeat this process infinitely often, we say the map $f$ is infinitely renormalizable. We note that every tent map $T_{a}$ with $a \in(1, \sqrt{2}]$ is renormalizable, and every tent map $T_{a}$ with $a \in(\sqrt{2}, 2]$ is non-renormalizable; no map in the tent family is infinitely renormalizable. A unimodal map $f$ is locally eventually onto (leo) provided that for every $\epsilon>0$ there exists $M \in \mathbb{N}$ such that, if $U$ is an interval with $|U|>\epsilon$ and if $n \geq M$, then $f^{n}(U)=\left[c_{2}, c_{1}\right]$. In the case where $a \in(\sqrt{2}, 2], T_{a}$ is leo; moreover, any renormalizable map is not leo.

Throughout this paper we use the notation $\langle a ; b\rangle$ to denote the interval with endpoints $a$ and $b$ when it is unclear whether $a<b$ or $b<a$. For each set $S$, the convex hull is denoted $[S]$.

2.2. Adding machines. Let $\alpha=\left\langle q_{1}, q_{2}, \ldots\right\rangle$ be a sequence of integers where each $q_{i} \geq 2$. Define $\Delta_{\alpha}$ to be the set of all sequences $\left(a_{1}, a_{2} \ldots\right)$ such that $0 \leq a_{i} \leq q_{i}-1$ for each $i$. Apply the metric $d_{\alpha}$ to $\Delta_{\alpha}$ by

$$
d_{\alpha}\left(\left(x_{1}, x_{2}, \ldots\right),\left(y_{1}, y_{2}, \ldots\right)\right)=\sum_{i=1}^{\infty} \frac{\delta\left(x_{i}, y_{i}\right)}{2^{i}}
$$


where $\delta\left(x_{i}, y_{i}\right)=0$ if $x_{i}=y_{i}$ and $\delta\left(x_{i}, y_{i}\right)=1$ otherwise. Addition on $\Delta_{\alpha}$ is defined as follows. Set

$$
\left(x_{1}, x_{2}, \ldots\right)+\left(y_{1}, y_{2}, \ldots\right)=\left(z_{1}, z_{2}, \ldots\right)
$$

where $z_{1}=x_{1}+y_{1} \bmod q_{1}$, and for each $j \geq 2, z_{j}=x_{j}+y_{j}+r_{j-1} \bmod q_{j}$ with $r_{j-1}=0$ if $x_{j-1}+y_{j-1}+r_{j-2}<q_{j-1}$ and $r_{j-1}=1$ otherwise (we set $\left.r_{0}=0\right)$. Define $f_{\alpha}: \Delta_{\alpha} \rightarrow \Delta_{\alpha}$ by

$$
f_{\alpha}\left(\left(x_{1}, x_{2}, \ldots\right)\right)=\left(x_{1}, x_{2}, x_{3}, \ldots\right)+(1,0,0, \ldots) .
$$

The dynamical system $f_{\alpha}: \Delta_{\alpha} \rightarrow \Delta_{\alpha}$ is the $\alpha$-adic adding machine map. Note that $f_{\alpha}$ is one-to-one and onto.

The following theorem provides a characterization for when a continuous map on a compact topological space is topologically conjugate to an adding machine. See also [7].

Theorem 2.1 ([3, Theorem 2.3]). Let $\alpha=\left\langle j_{1}, j_{2}, \ldots\right\rangle$ be a sequence of integers with $j_{i} \geq 2$ for each $i$. Let $m_{i}=j_{1} j_{2} \cdots j_{i}$ for each $i$. Let $f: X \rightarrow X$ be a continuous map of a compact topological space $X$. Then $f$ is topologically conjugate to $f_{\alpha}$ if and only if the following hold:

(1) For each positive integer $i$, there is a cover $\mathcal{P}_{i}$ of $X$ consisting of $m_{i}$ pairwise disjoint, nonempty, clopen sets which are cyclically permuted by $f$.

(2) For each positive integer $i, \mathcal{P}_{i+1}$ partitions $\mathcal{P}_{i}$.

(3) If mesh $\left(\mathcal{P}_{i}\right)$ denotes the maximum diameter of an element of the cover $\mathcal{P}_{i}$, then mesh $\left(\mathcal{P}_{i}\right) \rightarrow 0$ as $i \rightarrow \infty$.

The following theorem from [4 proves the existence of SAMs.

Theorem 2.2 ([4, Theorem 3.1]). Let $\alpha=\left\langle p_{1}, p_{2}, \cdots\right\rangle$ be a sequence of integers greater than 1 . The set of parameters $s$, such that for the tent map $f_{s}$ the restriction of $f_{s}$ to the closure of the orbit of $c=1 / 2$ is topologically conjugate to $f_{\alpha}: \Delta_{\alpha} \rightarrow \Delta_{\alpha}$, is dense in $[\sqrt{2}, 2]$.

Given $f \in \mathcal{A}$, there exists a nested hierarchy $\mathcal{H}=\left\{\mathcal{P}_{n} \mid n \in \mathbb{N}\right\}$ of partitions of $\omega(c)$ satisfying conditions (1), (2), and (3) of Theorem 2.1. For ease of notation, the following hold for each $n \in \mathbb{N}$ :

- The collection $\mathcal{P}_{n}$ equals $\left\{P_{n, i}\right\}_{i=0}^{\left|\mathcal{P}_{n}\right|-1}$.

- The unique element of $\mathcal{P}_{n}$ containing $c$ is denoted $P_{n, 0}$.

- For each $i=1, \ldots,\left|\mathcal{P}_{n}\right|-1, P_{n, i}=f\left(P_{n, i-1}\right)$.

Note that $f\left(P_{n,\left|\mathcal{P}_{n}\right|-1}\right)=P_{n, 0}$ and $c_{i} \in P_{n, i}$ for each $i=1, \ldots,\left|\mathcal{P}_{n}\right|-1$.

A set $S \subset[0,1]$ is said to straddle $x \in[0,1]$ if $S$ contains points lying on both sides of $x$ but does not contain $x$.

Proposition 2.3. Let $f \in \mathcal{A}$ be a symmetric tent map. Then for each $n \in \mathbb{N}$ the partition $\mathcal{P}_{n}$ of $\omega(c)$ from $\mathcal{H}$ contains an element straddling $c$. 
Proof. It suffices to assume $f$ is leo (i.e., has slope greater than $\sqrt{2}$ ). Fix $n \in \mathbb{N}$ and assume that no element of $\mathcal{P}_{n}$ straddles $c$. Then for each $1 \leq i \leq$ $\left|\mathcal{P}_{n}\right|-1,\left[P_{n, i}\right]$ lies entirely on one side of $c$. Hence $\left.f\right|_{\left[P_{n, i}\right]}$ is one-to-one and onto $\left[P_{n, i+1}\right]$ for each $1 \leq i \leq\left|\mathcal{P}_{n}\right|-2$, and $f\left(\left[P_{n,\left|\mathcal{P}_{n}\right|-1}\right]\right)=\left[P_{n, 0}\right]$ in a one-toone manner. Since $f\left(\left[P_{n, 0}\right]\right)=\left[P_{n, 1}\right]$, it follows that $f^{k \cdot\left|\mathcal{P}_{n}\right|}\left(\left[P_{n, 0}\right]\right)=\left[P_{n, 0}\right]$ for all $k \in \mathbb{N}$. As $f$ is locally eventually onto, this is a contradiction. Thus every partition $\mathcal{P}_{n}$ of $\omega(c)$ must contain an element straddling $c$.

In contrast to Proposition 2.3 , given an infinitely renormalizable logistic map $f \in \mathcal{A}$ and a partition $\mathcal{P}_{n} \in \mathcal{H}$ the collection $\left\{\left[P_{n, k}\right]\right\}_{k=0}^{\left|\mathcal{P}_{n}\right|-1}$ of convex hulls is pairwise disjoint, i.e., no element from $\mathcal{P}_{n}$ straddles $c$.

2.3. Inverse limit spaces. Given a continuum (compact connected metric space) $I$ and a continuous map $f: I \rightarrow I$, the associated inverse limit space $(I, f)$ is defined by

$$
(I, f)=\left\{x=\left(x_{0}, x_{1}, \ldots\right) \mid x_{n} \in I \text { and } f\left(x_{n+1}\right)=x_{n} \text { for all } n \in \mathbb{N}\right\}
$$

and has metric

$$
d(x, y)=\sum_{i=0}^{\infty} \frac{\left|x_{i}-y_{i}\right|}{2^{i}} .
$$

The map $\hat{f}:(I, f) \rightarrow(I, f)$ given by $\hat{f}\left(\left(x_{0}, x_{1}, \ldots\right)\right)=\left(f\left(x_{0}\right), x_{0}, x_{1}, \ldots\right)$ is called the induced homeomorphism on $(I, f)$. For $x \in(I, f), \pi_{i}(x)=x_{i}$ denotes the $i$ th projection of $x$. The backward itinerary of a point $x \in(I, f)$ is defined coordinatewise by $\mathcal{I}_{i}(x)$, where $\mathcal{I}_{i}(x)=1$ if $x_{i}>c, \mathcal{I}_{i}(x)=0$ if $x_{i}<c$, and $\mathcal{I}_{i}(x)=*$ if $x_{i}=c$.

As in [6], for each $x \in(I, f)$ such that $x_{i} \neq c$ for all $i>0$, set

$$
\begin{array}{r}
\tau_{R}(x)=\sup \left\{n \geq 1 \mid \mathcal{I}_{n-1}(x) \mathcal{I}_{n-2}(x) \cdots \mathcal{I}_{1}(x)=e_{1} e_{2} \cdots e_{n-1}\right. \text { and } \\
\left.\quad \#\left\{1 \leq i \leq n \mid e_{i}=1\right\} \text { is even }\right\}
\end{array}
$$

and

$$
\begin{array}{r}
\tau_{L}(x)=\sup \left\{n \geq 1 \mid \mathcal{I}_{n-1}(x) \mathcal{I}_{n-2}(x) \cdots \mathcal{I}_{1}(x)=e_{1} e_{2} \cdots e_{n-1}\right. \text { and } \\
\left.\#\left\{1 \leq i \leq n \mid e_{i}=1\right\} \text { is odd }\right\} .
\end{array}
$$

Note that in general, $\tau_{L}(x)$ and/or $\tau_{R}(x)$ can be infinite. For each $x \in(I, f)$, set $\Gamma(x)=\left\{y \in(I, f) \mid \mathcal{I}_{i}(y)=\mathcal{I}_{i}(x)\right.$ for all $\left.i \geq 1\right\}$.

As we focus on unimodal bonding maps, our inverse limit spaces are atriodic (i.e., contain no homeomorphic copies of the letter $\mathrm{Y}$ ); hence we may use the following definition. A point $x \in(I, f)$ is an endpoint of $(I, f)$ provided for every pair $A$ and $B$ of subcontinua of $(I, f)$ with $x \in A \cap B$, either $A \subset B$ or $B \subset A$. Barge and Martin characterize endpoints using the concept of $\epsilon$-crooked. Namely, if $f:[c, d] \rightarrow[a, b]$ is a continuous surjection (not necessarily unimodal), $p \in[c, d]$, and $\epsilon>0$, then $f$ is $\epsilon$-crooked with 
respect to $p$ provided $p$ does not separate $f^{-1}([a, a+\epsilon])$ from $f^{-1}([b-\epsilon, b])$ in $[c, d]$.

TheOREm 2.4 ([2, Theorem 1.4]). Let I be a closed interval, and let $f: I \rightarrow I$ be continuous. Then $p=\left(p_{0}, p_{1}, \ldots\right)$ is an endpoint of $(I, f)$ if and only if for each positive integer $i$, each interval $J_{i}$ with $p_{i} \in \operatorname{int}\left(J_{i}\right)$, and each $\epsilon>0$, there is a positive integer $N$ such that if $p_{i+N} \in J_{i+N}$ and $f^{N}\left(J_{i+N}\right)=J_{i}$, then $f^{N}$ is $\epsilon$-crooked with respect to $p_{i+N}$.

3. Endpoints and embedded adding machines. In Theorem 3.4 we characterize the endpoints of $(I, f)$ where $f \in \mathcal{A}$. In Theorem 3.5 and Corollary 3.6 we precisely define the collection of all endpoints of $(I, f)$ where $f$ is an infinitely renormalizable logistic map. Here and throughout the remainder of the paper, $I$ denotes the core $\left[c_{2}, c_{1}\right]$.

Definition 3.1. For a unimodal map $f$, we define $\mathcal{E}_{f}:=\left\{\left(x_{0}, x_{1}, \ldots\right) \in\right.$ $(I, f) \mid x_{i} \in \omega(c, f)$ for all $\left.i \in \mathbb{N}\right\}$. When the map $f$ is clearly understood in the context, we simply denote this set $\mathcal{E}$.

Lemma 3.2. Let $f$ be a unimodal map with $\mathcal{K}(f) \neq 10^{\infty}$ and suppose $x=\left(x_{0}, x_{1}, \ldots\right) \in(I, f) \backslash \mathcal{E}$. Then $x$ is not an endpoint of $(I, f)$.

Proof. If $c$ is not recurrent and $\mathcal{K}(f) \neq 10^{\infty}$, then $(I, f)$ has no endpoints. We thus assume that $c$ is recurrent. Let $x \in(I, f) \backslash \mathcal{E}$. We may choose $i \in \mathbb{N}$ such that $x_{i} \notin \omega(c, f)$ and since $c$ is recurrent we have $\left\{c_{n} \mid n \geq 0\right\} \subset \omega(c, f)$. Thus we may choose an interval $J_{i}$ with $x_{i}$ in the interior of $J_{i}$ and such that $J_{i} \cap \omega(c, f)=\emptyset$. Fix $\epsilon>0$ and $N \in \mathbb{N}$. Let $J_{i+N} \ni x_{i+N}$ be such that $f^{N}\left(J_{i+N}\right)=J_{i}$. Since $\left\{c_{n} \mid n \geq 0\right\} \cap J_{i}=\emptyset$, we see that $f^{N} \mid J_{i+N}$ is monotone and hence $f^{N}$ is not $\epsilon$-crooked with respect to $x_{i+N}$.

If no such $J_{i+N}$ exists, use the continuity of $f$ to obtain an interval $V \ni x_{i+N}$ such that $f^{N}(V) \subset J_{i}$. Set $J_{i}=f^{N}(V)$ and $J_{i+N}=V$. Then $f^{N}$ maps $J_{i+N}$ monotonically onto $J_{i}$. It follows from Theorem 2.4 that $x$ is not an endpoint of $(I, f)$.

Bruin [6] provides the following characterization for endpoints.

Proposition 3.3 ([6, Proposition 2]). Let $f$ be a unimodal map and $x \in(I, f)$ be such that $x_{i} \neq c$ for all $i \geq 0$. Then $x$ is an endpoint of $(I, f)$ if and only if $\tau_{R}(x)=\infty$ and $x_{0}=\sup \pi_{0}(\Gamma(x))\left(\right.$ or $\tau_{L}(x)=\infty$ and $\left.x_{0}=\inf \pi_{0}(\Gamma(x))\right)$.

Theorem 3.4 demonstrates that fewer conditions suffice in the case where $f \in \mathcal{A}$.

Theorem 3.4. Let $f \in \mathcal{A}$ and $x \in \mathcal{E}$ be such that $x_{i} \neq c$ for all $i \geq 0$. Then $x$ is an endpoint of $(I, f)$ if and only if $\tau_{R}(x)=\infty$ or $\tau_{L}(x)=\infty$. 
Proof. The forward direction follows immediately from Proposition 3.3 . We thus assume that $x$ is not an endpoint of $(I, f)$. Then there exist subcontinua $A$ and $B$ of $(I, f)$ such that $x \in A \cap B, A \backslash B \neq \emptyset$, and $B \backslash A \neq \emptyset$. For all $i$, we set $A_{i}=\pi_{i}(A)$ and $B_{i}=\pi_{i}(B)$. We set $J_{i}=A_{i} \cup B_{i}$ and since $x_{i} \in A_{i} \cap B_{i}$, it follows that $J_{i}$ is an interval in $I$. Let $J_{i}=\left[a_{i}, b_{i}\right]$. Since $A \backslash B \neq \emptyset$ and $B \backslash A \neq \emptyset$, there exists an $i \in \mathbb{N}$ such that whenever $j \geq i$, then $A_{j} \backslash B_{j} \neq \emptyset$ and $B_{j} \backslash A_{j} \neq \emptyset$. Fix this particular $i \in \mathbb{N}$ and choose $\epsilon>0$ small such that $\left[a_{i}, a_{i}+\epsilon\right]$ lies in one of $A_{i} \backslash B_{i}$ or $B_{i} \backslash A_{i}$ and $\left[b_{i}-\epsilon, b_{i}\right]$ lies in the other. Then for each $\alpha \in \mathbb{N}, f^{\alpha}\left(A_{i+\alpha}\right)=A_{i}, f^{\alpha}\left(B_{i+\alpha}\right)=B_{i}$, and $x_{i+\alpha} \in A_{i+\alpha} \cap B_{i+\alpha}$. Thus $x_{i+\alpha}$ separates $f^{-\alpha}\left(\left[a_{i}, a_{i}+\epsilon\right]\right)$ and $f^{-\alpha}\left(\left[b_{i}-\epsilon, b_{i}\right]\right)$ for all $\alpha \in \mathbb{N}$. That is, for each $\alpha \in \mathbb{N}, f^{\alpha}: J_{i+\alpha} \rightarrow J_{i}$ fails to be $\epsilon$-crooked with respect to $x_{i+\alpha}$.

Let $\mathcal{H}=\left\{\mathcal{P}_{m}\right\}_{m \geq 1}$ be a nested hierarchy of partitions of $\omega(c)$. Because $\operatorname{mesh}\left(\mathcal{P}_{m}\right) \rightarrow 0$, there exists $N \in \mathbb{N}$ such that the element $P_{N, j}$ of $\mathcal{P}_{N}$ containing $x_{i}$ lies completely in $\left(a_{i}+\epsilon, b_{i}-\epsilon\right)$, and moreover $j \neq 0$.

Thus $x_{i+j} \in P_{N, 0}$. Note that $f^{j}$ is not monotone on $\left\langle x_{i+j} ; c\right\rangle$. If $f^{j}$ were monotone on that interval, then $f^{j}\left(J_{i+j} \backslash\left\langle c ; x_{i+j}\right\rangle\right)=J_{i}$, since $f^{j}$ is symmetric about $c$ and $c_{j} \in\left(a_{i}+\epsilon, b_{i}-\epsilon\right)$. But then $x_{i+j}$ would not separate $f^{-j}\left(\left[a_{i}, a_{i}+\epsilon\right]\right)$ and $f^{-j}\left(\left[b_{i}-\epsilon, b_{i}\right]\right)$, a contradiction.

Note that as $x_{i} \neq c_{k}$ for any $k \geq 0$, there exists $M>N$ such that $x_{i} \notin P_{M, j}$ (else $x_{i}=c_{j}$ ). Hence we may always find $M>N$ and $k>j$ such that $x_{i} \in P_{M, k}$. Now suppose that $\mathcal{P}_{M}$ is fixed with $M>N$ and $x_{i} \in P_{M, k}$. Then we may find $n>k$ such that whenever $I(f(p))$ begins $e_{1} \cdots e_{n}$, then $p \in\left[P_{M, 0}\right]$.

Suppose either $\tau_{R}(x)=\infty$ or $\tau_{L}(x)=\infty$. Then there exists $L>n$ such that $\mathcal{I}_{L+i-1}(x) \cdots \mathcal{I}_{i}(x)=e_{1} \cdots e_{L}$. Hence $x_{i+L} \in P_{M, 0}, c_{L} \in P_{M, k}$, and $f^{L}$ is monotone on $\left\langle x_{i+L} ; c\right\rangle$. Consider $J_{i+L}$. If $J_{i+L} \ni c$, then the monotonicity of $f^{L}$ on the interval $\left\langle x_{i+L} ; c\right\rangle$ forces $f^{L}: J_{i+L} \rightarrow J_{i}$ to be $\epsilon$-crooked with respect to $x_{i+L}$, a contradiction. If $c \notin J_{i+L}$, then as $x_{i+L} \in \operatorname{int}\left(J_{i+L}\right)$ and $f^{L}$ maps $\left\langle x_{i+L} ; c\right\rangle$ monotonically into $\left(a_{i}+\epsilon, b_{i}-\epsilon\right), f^{L}: J_{i+L} \rightarrow J_{i}$ will again be $\epsilon$-crooked with respect to $x_{i+L}$. Hence $\tau_{R}(x)<\infty$ and $\tau_{L}(x)<\infty$.

In Theorem 3.4 we assume $x_{i} \neq c$ for all $i \geq 0$. In the case where $x_{i}=c$ for some $i$, we note that this can occur for at most one $i$, as $c$ is nonperiodic. Hence if $x_{i}=c$ for some $i \geq 0$, set $y=\hat{f}^{-(i+1)}(x)$. Then $x$ is an endpoint of $(I, f)$ if and only if $y$ is an endpoint of $(I, f)$. As $y_{i} \neq c$ for all $i \geq 0$ and $y \in \mathcal{E}$, we may check whether $y$ is an endpoint using Theorem 3.4 .

THEOREM 3.5. Let $f$ be an infinitely renormalizable logistic map and let $x \in \mathcal{E}$ be such that $x_{i} \neq c$ for all $i \geq 0$. Then $\tau_{R}(x)=\infty$ or $\tau_{L}(x)=\infty$.

Proof. As $f$ is an infinitely renormalizable logistic map, it follows that $f \in \mathcal{A}$. Since $x \in \mathcal{E}$ with $x_{i} \neq c$ for all $i \geq 0$, we have $x_{0} \in \omega(c)$ and $x_{0} \neq c_{j}$ for all $j \geq 0$. Let $\mathcal{H}=\left\{\mathcal{P}_{n}\right\}_{n \geq 1}$. Suppose $x_{0} \in P_{n, j}$ for some 
$n, j \in \mathbb{N}$. Then there exist $m>n$ and $k>j$ such that $x_{0} \in P_{m, k}$ (else $x_{0} \in P_{N, j}$ for all $N \geq n$, implying $x_{0}=c_{j}$, a contradiction). As the collection $\left\{\left[P_{n, i}\right]\right\}_{i=0}^{\left|\mathcal{P}_{n}\right|-1}$ of convex hulls is pairwise disjoint for each $n \in \mathbb{N}$, all points $y \in$ $\mathcal{E}$ with $y_{0} \in\left[P_{m, k}\right]$ have backward itineraries with $\mathcal{I}_{k-1}(y) \mathcal{I}_{k-2}(y) \cdots \mathcal{I}_{1}(y)=$ $e_{1} e_{2} \cdots e_{k-1}$. Note that $m, k \in \mathbb{N}$ may be chosen arbitrarily large such that $x_{0} \in P_{m, k}$; hence at least one of $\tau_{R}(x)$ or $\tau_{L}(x)$ is infinite.

COROLlary 3.6. Let $f$ be an infinitely renormalizable logistic map. Then $\mathcal{E}$ is precisely the collection of endpoints of $(I, f)$.

The following definitions and basic results are used in Section 4 .

Definition 3.7. Given a unimodal map $f$ and $j \in \mathbb{N}$, we define $V_{j}$ to be the maximal open interval containing $c_{1}$ for which $f^{j}$ is monotone on $V_{j}$. For $x \in(I, f)$ set $A_{x}=\left\{k \mid x_{k} \in V_{k}\right\}$.

Clearly $V_{j} \subseteq V_{k}$ for all $j \geq k$. For $x \in(I, f)$, if $x_{j} \in V_{j}$, then the itineraries of $x_{j}$ and $c_{1}$ agree for at least $j-1$ iterates under $f$; thus $x_{1}$ and $c_{j}$ must lie on the same side of $c$. If $x_{j} \in V_{j-1} \backslash V_{j}$, then $x_{1}$ and $c_{j}$ lie on opposite sides of $c$. In both of these cases, as $x_{j} \in V_{j-1}$, it follows that $x_{j-i}$ and $c_{i+1}$ lie on the same side of $c$ for $i=0, \ldots, j-2$.

Proposition 3.8. Let $f$ be a unimodal map and $x \in \mathcal{E}$ be such that $x_{i} \neq c$ for all $i \geq 0$. Then $\tau_{R}(x)<\infty$ and $\tau_{L}(x)<\infty$ if and only if $\left|A_{x}\right|<\infty$.

Proof. Assume $\left|A_{x}\right|<\infty$. Then there exists a maximal $k$ such that $x_{k} \in V_{k}$, and thus for all $j>k$ there exists $0 \leq m_{j} \leq j-1$ such that $c_{m_{j}+1}$ and $x_{j-m_{j}}$ lie on opposite sides of $c$. Hence $\tau_{R}(x)<\infty$ and $\tau_{L}(x)<\infty$.

Now assume $\left|A_{x}\right|=\infty$. Then there exist infinitely many $k$ such that $x_{k} \in V_{k}$ and $\mathcal{I}_{k}(x) \mathcal{I}_{k-1}(x) \cdots \mathcal{I}_{1}(x)=e_{1} e_{2} \cdots e_{k}$. Hence either $\tau_{R}(x)=\infty$ or $\tau_{L}(x)=\infty$.

Corollary 3.9. Let $f \in \mathcal{A}$ and $x \in \mathcal{E}$ be such that $x_{i} \neq c$ for all $i \geq 0$. Then $x$ is an endpoint of $(I, f)$ if and only if $\left|A_{x}\right|=\infty$.

4. Non-endpoints and strange adding machines. In this section we use the SAM scheme provided in [4] to prove the following theorem.

THEOREM 4.1. For each symmetric tent map $g$ constructed from an SAM scheme as in [4] with $\left.g\right|_{\omega(c)}$ topologically conjugate to an adding machine, the set of endpoints of $(I, g)$ is a proper subset of $\mathcal{E}$.

We next provide an outline for the construction of a symmetric tent map $g$ obtained by an SAM scheme [4], define a specific nested hierarchy of partitions that exists for the strange adding machine embedded in $g$, and then use this hierarchy to locate non-endpoints lying in $\mathcal{E}$. We recall the following definitions and results from [4]. 
Definition 4.2 ([4, Definition 2.1]). Given a symmetric tent map $f$, an $S A M$ scheme for $f$ of length $s+t+1$ is a collection $\mathcal{C}_{1}$ of disjoint, closed subintervals of $[0,1]$,

$$
\mathcal{C}_{1}=\left\{L_{2}, L_{1}, R_{1}, R_{2}, A_{1}, \ldots, A_{s}, B_{1}, \ldots, B_{s}, Y_{1}, \ldots, Y_{t}\right\}
$$

such that each of the following holds:

- $L_{2}<L_{1}<\{c\}<R_{1}<R_{2}$ (i.e., $L_{2}$ lies to the left of $L_{1}$ ).

- For each $i=1, \ldots, s, A_{i} \cap\left[L_{2} \cup R_{2}\right]=\emptyset$ and $B_{i} \cap\left[L_{2} \cup R_{2}\right]=\emptyset$. Also, for each $i=1, \ldots, t, Y_{i} \cap\left[L_{2} \cup R_{2}\right]=\emptyset$.

- $f\left(L_{1}\right)=f\left(R_{1}\right)=A_{1}$ and $f\left(L_{2}\right)=f\left(R_{2}\right)=B_{1}$.

- For each $i=1, \ldots, s-1, f\left(A_{i}\right)=A_{i+1}$ and $f\left(B_{i}\right)=B_{i+1}$.

- $f\left(A_{s}\right)=f\left(B_{s}\right)=Y_{1}$.

- For each $i=1, \ldots, t-1, f\left(Y_{i}\right)=Y_{i+1}$.

- The set $A_{s} \cup B_{s}$ straddles $c$, but for each $i=1, \ldots, s-1$ the set $A_{i} \cup B_{i}$ does not straddle $c$.

- $f\left(Y_{t}\right)=\left[L_{2} \cup R_{2}\right]$.

Definition 4.3 ([4, Definition 2.2]). Let $f$ be a symmetric tent map and suppose

$$
\mathcal{C}_{1}=\left\{L_{2}^{1}, L_{1}^{1}, R_{1}^{1}, R_{2}^{1}, A_{1}^{1}, \ldots, A_{s_{1}}^{1}, B_{1}^{1}, \ldots, B_{s_{1}}^{1}, Y_{1}^{1}, \ldots, Y_{t_{1}}^{1}\right\}
$$

and

$$
\mathcal{C}_{2}=\left\{L_{2}^{2}, L_{1}^{2}, R_{1}^{2}, R_{2}^{2}, A_{1}^{2}, \ldots, A_{s_{2}}^{2}, B_{1}^{2}, \ldots, B_{s_{2}}^{2}, Y_{1}^{2}, \ldots, Y_{t_{2}}^{2}\right\}
$$

are SAM schemes for $f$. Then $\mathcal{C}_{2}$ is a refinement of $\mathcal{C}_{1}$ provided the following hold.

- Each interval in the collection $\mathcal{C}_{2}$ is a subset of an interval in the collection $\mathcal{C}_{1}$.

- $L_{2}^{2} \subset L_{1}^{1}, L_{1}^{2} \subset L_{1}^{1}, R_{1}^{2} \subset R_{1}^{1}$, and $R_{2}^{2} \subset R_{1}^{1}$.

- For each $i=1, \ldots, s_{2}-1, A_{i}^{2} \cup B_{i}^{2}$ is contained in one of the intervals in the collection $\mathcal{C}_{1}$.

- One of the two intervals $A_{s_{2}}^{2}, B_{s_{2}}^{2}$ is contained in $L_{2}^{1}$ and the other interval is contained in $R_{2}^{1}$.

We now begin with an arbitrary symmetric tent map $f$ with slope greater than $\sqrt{2}$ and let $\alpha=\left\langle p_{1}, p_{2}, \ldots\right\rangle$ be a sequence of integers with $p_{i} \geq 2$ for all $i$. As is shown in [4], we may let $\mathcal{C}_{1}$ be an SAM scheme for $f$ of length $p_{1} \cdots p_{d}$ for some $d \in \mathbb{N}$. Again as in [4], we may obtain a sequence $\left\{\mathcal{C}_{n}\right\}_{n \geq 1}$ of refinements such that $\mathcal{C}_{2}$ has length $p_{1} \cdots p_{d+1}, \mathcal{C}_{3}$ has length $p_{1} \cdots p_{d+2}$, etc. For each $n \in \mathbb{N}$, denote

$$
\mathcal{C}_{n}=\left\{L_{2}^{n}, L_{1}^{n}, R_{1}^{n}, R_{2}^{n}, A_{1}^{n}, \ldots, A_{s_{n}}^{n}, B_{1}^{n}, \ldots, B_{s_{n}}^{n}, Y_{1}^{n}, \ldots, Y_{t_{n}}^{n}\right\} .
$$

By the proof of [4, Lemma 2.5], the refinement $\mathcal{C}_{n+1}$ of $\mathcal{C}_{n}$ is chosen such that $A_{1}^{n+1} \subset A_{1}^{n}, B_{1}^{n+1} \subset A_{1}^{n}$, and if we set $S=M B_{1}^{n} \cdots B_{s_{n}}^{n} Y_{1}^{n} \cdots Y_{t_{n}}^{n}$ 
where $M \in\left\{L_{2}^{n}, R_{2}^{n}\right\}$, then $A_{1}^{n+1}$ and $B_{1}^{n+1}$ both "permute through" (by the map $f$ ) the elements of $\mathcal{C}_{n}$ in the following order:

$$
A_{1}^{n} \cdots A_{s_{n}}^{n} Y_{1}^{n} \cdots Y_{t_{n}}^{n} \underbrace{S \cdots S}_{p_{d^{\prime}+1}-2 \text { times }}\left(L_{2}^{n} \cup R_{2}^{n}\right) B_{1}^{n} \cdots B_{s_{n}}^{n} Y_{1}^{n} \cdots Y_{t_{n}}^{n} .
$$

Here $p_{1} \cdots p_{d^{\prime}}=s_{n}+t_{n}+1$ is the length of $\mathcal{C}_{n}$ and $p_{1} \cdots p_{d^{\prime}+1}=s_{n+1}+t_{n+1}+1$ is the length of $\mathcal{C}_{n+1}$. Note that $s_{n+1}=\left(p_{d^{\prime}+1}-1\right)\left(t_{n}+s_{n}+1\right)$ and $t_{n+1}=$ $t_{n}+s_{n}$. The only disagreement in the "permutations" of $A_{1}^{n+1}$ and $B_{1}^{n+1}$ occurs in the $s_{n+1}$ th position, denoted above by $L_{2}^{n} \cup R_{2}^{n}$, where one traces through $L_{2}^{n}$ and the other traces through $R_{2}^{n}$. It follows that $Y_{1}^{n+1} \subset B_{1}^{n}$ and $Y_{s_{n}+1}^{n+1} \subset Y_{1}^{n}$.

As in [4, for $n \in \mathbb{N}$, set $\mathbf{C}_{n}$ to be the collection of all $x \in[0,1]$ such that the forward orbit of $x$ under $f$ lies in $\bigcup_{J \in \mathcal{C}_{n}} J$. Note that each point in the forward orbit of $x \in \mathbf{C}_{n}$ lies in exactly one element of $\mathcal{C}_{n}$. Let $\mathbf{C}=\bigcap_{n \geq 1} \mathbf{C}_{n}$.

REMARK 4.4. Given $n \in \mathbb{N}$ and an SAM scheme $\mathcal{C}_{n}$, set $M^{n}=\left[L_{2}^{n} \cup R_{2}^{n}\right]$ and use this set to replace the sets $L_{2}^{n}, L_{1}^{n}, R_{1}^{n}$, and $R_{2}^{n}$. This new collection of sets will be denoted

$$
\mathcal{D}_{n}=\left\{M^{n}, A_{1}^{n}, \ldots, A_{s_{n}}^{n}, B_{1}^{n}, \ldots, B_{s_{n}}^{n}, Y_{1}^{n}, \ldots, Y_{t_{n}}^{n}\right\} .
$$

Thus, for each $n \in \mathbb{N}, f$ acts on the collection of points $\mathbf{C}$ in the following way.

- $f\left(M^{n} \cap \mathbf{C}\right)=\left(A_{1}^{n} \cup B_{1}^{n}\right) \cap \mathbf{C}$.

- For each $i=1, \ldots, s_{n}-1, f\left(\left(A_{i}^{n} \cup B_{i}^{n}\right) \cap \mathbf{C}\right)=\left(A_{i+1}^{n} \cup B_{i+1}^{n}\right) \cap \mathbf{C}$.

- $f\left(\left(A_{s_{n}}^{n} \cup B_{s_{n}}^{n}\right) \cap \mathbf{C}\right)=Y_{1}^{n} \cap \mathbf{C}$.

- For each $i=1, \ldots, t_{n}-1, f\left(Y_{i}^{n} \cap \mathbf{C}\right)=Y_{i+1}^{n} \cap \mathbf{C}$.

- $f\left(Y_{t_{n}}^{n} \cap \mathbf{C}\right)=M^{n} \cap \mathbf{C}$.

As in [4] we identify an interval $J$, a natural projection $\varphi:[0,1] \rightarrow J$, and a unimodal map $g: J \rightarrow J$ such that $g(\varphi(x))=\varphi(f(x))$ for all $x \in \mathbf{C}$. As $g: J \rightarrow J$ is conjugate to a symmetric tent map, we thus consider $g$ to be a symmetric tent map with turning point $c$. Note that the map $\varphi$ preserves order; that is, if $A<B$ with both $A, B$ in $\mathcal{D}_{n}$ for some $n$, then $\varphi(A)<\varphi(B)$ where both $\varphi(A)$ and $\varphi(B)$ are intervals in $J$. By the construction of $g, \varphi(\mathbf{C})=\omega(c, g)$ and $\left.g\right|_{\omega(c)}$ is topologically conjugate to $g_{\alpha}: \Delta_{\alpha} \rightarrow \Delta_{\alpha}$ (see [4]).

We now use the SAM scheme, $\varphi$, and $g$ from above to obtain a nested hierarchy $\left\{\mathcal{P}_{n}^{\varphi}\right\}_{n \geq 1}$ of partitions of $\omega(c, g)$ such that for each $n \in \mathbb{N}$, the $s_{n}+t_{n}+1$ elements of $\mathcal{P}_{n}^{\varphi}$ are labeled as follows:

- $P_{n, 0}=\varphi\left(M^{n} \cap \mathbf{C}\right)$.

- $P_{n, i}=\varphi\left(\left(A_{i}^{n} \cup B_{i}^{n}\right) \cap \mathbf{C}\right)$ for $i=1, \ldots, s_{n}$.

- $P_{n, i}=\varphi\left(Y_{i-s_{n}}^{n} \cap \mathbf{C}\right)$ for $i=s_{n}+1, \ldots, s_{n}+t_{n}$. 
Proposition 4.5. The hierarchy of partitions $\left\{\mathcal{P}_{n}^{\varphi}\right\}_{n \geq 1}$ satisfies the three conditions of Theorem 2.1.

Proof. Let $g$ be as above. As $g(\varphi(x))=\varphi(f(x))$ for all $x \in \mathbf{C}$, by Remark 4.4 we have the following for each $n \in \mathbb{N}$ :

- $g\left(P_{n, 0}\right)=g\left(\varphi\left(M^{n} \cap \mathbf{C}\right)\right)=\varphi\left(f\left(M^{n} \cap \mathbf{C}\right)\right)=\varphi\left(\left(A_{1}^{n} \cup B_{1}^{n}\right) \cap \mathbf{C}\right)=P_{n, 1}$.

- For each $i=1, \ldots, s_{n}-1, g\left(P_{n, i}\right)=g\left(\varphi\left(\left(A_{i}^{n} \cup B_{i}^{n}\right) \cap \mathbf{C}\right)\right)$ $=\varphi\left(f\left(\left(A_{i}^{n} \cup B_{i}^{n}\right) \cap \mathbf{C}\right)\right)=\varphi\left(\left(A_{i+1}^{n} \cup B_{i+1}^{n}\right) \cap \mathbf{C}\right)=P_{n, i+1}$.

- $g\left(P_{n, s_{n}}\right)=g\left(\varphi\left(\left(A_{s_{n}}^{n} \cup B_{s_{n}}^{n}\right) \cap \mathbf{C}\right)\right)=\varphi\left(f\left(\left(A_{s_{n}}^{n} \cup B_{s_{n}}^{n}\right) \cap \mathbf{C}\right)\right)$ $=\varphi\left(Y_{1}^{n} \cap \mathbf{C}\right)=P_{n, s_{n}+1}$.

- For each $i=1, \ldots, t_{n}-1, g\left(P_{n, s_{n}+i}\right)=g\left(\varphi\left(Y_{i}^{n} \cap \mathbf{C}\right)\right)=\varphi\left(f\left(Y_{i}^{n} \cap \mathbf{C}\right)\right)=$ $\varphi\left(Y_{i+1}^{n} \cap \mathbf{C}\right)=P_{n, s_{n}+i+1}$.

- $g\left(P_{n, s_{n}+t_{n}}\right)=g\left(\varphi\left(Y_{t_{n}}^{n} \cap \mathbf{C}\right)\right)=\varphi\left(f\left(Y_{t_{n}}^{n} \cap \mathbf{C}\right)\right)=\varphi\left(M^{n} \cap \mathbf{C}\right)=P_{n, 0}$.

As the intervals in $\mathcal{D}_{n}$ are pairwise disjoint and $\varphi$ preserves order on $\mathcal{D}_{n}$, it follows that the elements of $\mathcal{P}_{n}^{\varphi}$ are pairwise disjoint. By the construction of $\mathbf{C}$ each element of $\mathcal{P}_{n}^{\varphi}$ is nonempty and clopen. It is shown in the proof of [4, Theorem 3.1] that $\operatorname{mesh}\left(\mathcal{P}_{n}^{\varphi}\right) \rightarrow 0$.

Since $\varphi$ is the natural projection and $\mathcal{D}_{n+1}$ refines $\mathcal{D}_{n}$ for each $n \in \mathbb{N}$, it follows that $\mathcal{P}_{n+1}^{\varphi}$ refines $\mathcal{P}_{n}^{\varphi}$.

Given a symmetric tent map $f$ and a sequence $\alpha=\left\langle p_{1}, p_{2}, \ldots\right\rangle$ of integers with each $p_{i} \geq 2$, we have produced a symmetric tent map $g$ such that $\left.g\right|_{\omega(c)}$ is topologically conjugate to the adding machine $g_{\alpha}: \Delta_{\alpha} \rightarrow \Delta_{\alpha}$. We also precisely described a nested hierarchy $\left\{\mathcal{P}_{n}^{\varphi}\right\}_{n \geq 1}$ of partitions that identify the adding machine embedded in $g$. Further, the construction in [4, Theorem 3.1] allows $g$ to be taken arbitrarily close to $f$.

We next establish that the set of endpoints of $(I, g)$ is a proper subset of $\mathcal{E}$. First a few observations. Fix $n \in \mathbb{N}$. Then $P_{n+1, s_{n+1}+s_{n}+1} \subseteq P_{n, s_{n}+1}$. By construction $c_{s_{n}} \in \varphi\left(A_{s_{n}}^{n} \cap \mathbf{C}\right)$ and the only element of $\mathcal{P}_{n}^{\varphi}$ straddling $c$ is $P_{n, s_{n}}$. Thus there exists an $x_{1} \in P_{n, s_{n}}$ such that $\left\langle x_{1} ; c_{s_{n}}\right\rangle \ni c$; namely, $x_{1} \in \varphi\left(B_{s_{n}}^{n} \cap \mathbf{C}\right)$ and if $x \in \mathcal{E}$ is such that $\pi_{1}(x)=x_{1}$, then $x_{s_{n}} \in V_{s_{n}-1} \backslash V_{s_{n}}$.

Proposition 4.6. Let $g$ be the symmetric tent map constructed above and $\left\{\mathcal{P}_{n}^{\varphi}\right\}_{n \geq 1}$ be the hierarchy of partitions of $\omega(c, g)$. Then there exists $x_{0} \in \omega(c, g)$ such that for all $n \in \mathbb{N}, x_{0} \in P_{n, s_{n}+s_{n-1}+\cdots+s_{1}+1}$, and there exists a unique point $x \in \mathcal{E}$ with $\pi_{0}(x)=x_{0}$. Moreover, $x_{i} \neq c$ for all $i \geq 0$ and $\left|A_{x}\right|<\infty$.

Proof. Recall that for each $n \in \mathbb{N}, P_{n, s_{n}+1} \supseteq P_{n+1, s_{n+1}+s_{n}+1}$. Further, by the construction of the partition $\mathcal{C}_{n+1}$ from $\mathcal{C}_{n}$, if $\mathcal{C}_{n}$ has length $p_{1} \cdots p_{d^{\prime}}$ and $\mathcal{C}_{n+1}$ has length $p_{1} \cdots p_{d^{\prime}+1}$, then $s_{n+1}=\left(p_{d^{\prime}+1}-1\right)\left(s_{n}+t_{n}+1\right)$. It thus follows, for each $n \in \mathbb{N}$, that $P_{n, s_{n}+1} \supseteq P_{n+1, s_{n+1}+s_{n}+1} \supseteq P_{n+2, s_{n+2}+s_{n+1}+s_{n}+1}$. 
We can extend this to obtain an infinite sequence of nested clopen sets

$$
P_{1, s_{1}+1} \supseteq P_{2, s_{2}+s_{1}+1} \supseteq P_{3, s_{3}+s_{2}+s_{1}+1} \supseteq \cdots,
$$

and as each set is nonempty, their intersection is nonempty. Thus there exists a point $x_{0} \in \varphi(\mathbf{C})=\omega(c, g)$ such that $x_{0} \in P_{n, s_{n}+s_{n-1}+\cdots+s_{1}+1}$ for all $n$, and hence $x_{0} \neq c_{i}$ for all $i \geq 0$. As $\left.g\right|_{\omega(c)}$ has a well-defined inverse (see Section 2), we may uniquely choose $x \in \mathcal{E}$ with $\pi_{0}(x)=x_{0}$ and $x_{i} \neq c$ for all $i \geq 0$.

For each $n \in \mathbb{N}$, if $x_{0} \in P_{n, s_{n}+s_{n-1}+\cdots+s_{1}+1}$ then $x_{1} \in P_{n+1, s_{n+1}+s_{n}+\cdots+s_{1}}$. As $P_{n+1, s_{n+1}+s_{n}+\cdots+s_{1}}=\varphi\left(Y_{s_{n}+\cdots+s_{1}}^{n+1} \cap \mathbf{C}\right)$ and $Y_{s_{n}}^{n+1} \subset B_{s_{n}}^{n}$, we see that $x_{s_{n}+\cdots+s_{1}} \in V_{s_{n}-1} \backslash V_{s_{n}}$ for all $n$. Hence $x_{s_{1}} \notin V_{s_{1}}$ and $s_{1} \notin A_{x}$. For each $n>s_{1}, n \in A_{x}$ only if $x_{n} \in P_{1,1}$; the only coordinates of $x$ lying in $P_{1,1}$ are of the form $x_{k \cdot\left(s_{1}+t_{1}+1\right)+s_{1}}$, where $k \in \mathbb{N}$. Thus it suffices to check only these coordinates to determine which values of $n$ are contained in the set $A_{x}$. By (4.1), $x_{k \cdot\left(s_{1}+t_{1}+1\right)+s_{1}} \in V_{s_{1}-1} \backslash V_{s_{1}}$ for all $k=0,1, \ldots, p_{d^{\prime}+1}-2$, where $\left(p_{d^{\prime}+1}-1\right)\left(s_{1}+t_{1}+1\right)=s_{2}$. Further, we have $x_{s_{2}+s_{1}} \in V_{s_{2}-1} \backslash V_{s_{2}}$. Thus $n \notin A_{x}$ for each $s_{1} \leq n \leq s_{2}+s_{1}$. Similarly, for each $n>s_{2}+s_{1}, n \in A_{x}$ only if $x_{n} \in P_{2,1}$. The only coordinates of $x$ lying in $P_{2,1}$ are of the form $x_{k \cdot\left(s_{2}+t_{2}+1\right)+s_{2}+s_{1}}$, where $k \in \mathbb{N}$. As above, it suffices to check only these coordinates when determining the values of $n$ lying in $A_{x}$; we conclude that $n \notin A_{x}$ for all $s_{2}+s_{1} \leq n \leq s_{3}+s_{2}+s_{1}$. Recursively we obtain $\left|A_{x}\right|<\infty$.

Proof of Theorem 4.1. Given the symmetric tent map $g$ constructed above and the nested hierarchy $\left\{\mathcal{P}_{n}^{\varphi}\right\}_{n \geq 1}$ of partitions of $\omega(c, g)$, there exists a point $x_{0} \in \omega(c, g)$ such that for all $n \in \mathbb{N}, x_{0} \in P_{n, s_{n}+s_{n-1}+\cdots+s_{1}+1}$. By Proposition 4.6 and Corollary 3.9, the point $x=\left(x_{0}, x_{1}, \ldots\right) \in \mathcal{E}$ is not an endpoint of $(I, g)$. By Lemma 3.2, the set of endpoints of $(I, g)$ is thus a proper subset of $\mathcal{E}$.

As $f$ was an arbitrary symmetric tent map with slope greater than $\sqrt{2}$ and $g$ can be constructed arbitrarily close to $f$, it follows that the set of parameters for which $\left.g\right|_{\omega(c)}$ is topologically conjugate to an adding machine with the set of endpoints of $(I, g)$ a proper subset of $\mathcal{E}$, is dense in $[\sqrt{2}, 2]$. Standard techniques then show that the set of parameters for which this is true is dense in $[1,2]$.

We close with the following observation.

Proposition 4.7. Let $g$ be a symmetric tent map constructed from an $S A M$ scheme as in [4] such that $\left.g\right|_{\omega(c)}$ is topologically conjugate to an adding machine. Then neither the set of endpoints of $(I, g)$ nor its complement in $\mathcal{E}$ is closed.

Proof. Let $x=\left(x_{0}, x_{1}, x_{2}, \ldots\right) \in \mathcal{E}$ be a non-endpoint of $(I, g)$ and $y=$ $\left(y_{0}, y_{1}, y_{2}, \ldots\right) \in \mathcal{E}$ be an endpoint of $(I, g)$. Thus $x_{0}, y_{0} \in \omega(c)$ and $\omega\left(y_{0}\right)=$ 
$\omega(c)$. Hence there exists an increasing sequence $\left\{k_{i}\right\}$ of positive integers such that $g^{k_{i}}\left(y_{0}\right) \rightarrow x_{0}$. Thus $\hat{g}^{k_{i}}(y) \rightarrow x$, and as $\hat{g}^{k_{i}}(y)$ is an endpoint of $(I, g)$ for all $i \in \mathbb{N}$, it follows that the set of endpoints of $(I, g)$ is not closed. A similar argument will show the complement of the endpoints in $\mathcal{E}$ is not closed.

\section{References}

[1] M. Barge, H. Bruin, and S. Štimac, The Ingram conjecture, preprint.

[2] M. Barge and J. Martin, Endpoints of inverse limit spaces and dynamics, in: Lecture Notes in Pure Appl. Math. 170, Dekker, 1995, 165-182.

[3] L. Block and J. Keesling, A characterization of adding machines maps, Topology Appl. 140 (2004), 151-161.

[4] L. Block, J. Keesling, and M. Misiurewicz, Strange adding machines, Ergodic Theory Dynam. Systems 26 (2006), 673-682.

[5] K. M. Brucks and H. Bruin, Topics From One-Dimensional Dynamics, Cambridge Univ. Press, 2004.

[6] H. Bruin, Planar embeddings of inverse limit spaces of unimodal maps, Topology Appl. 96 (1999), 191-208.

[7] J. Buescu and I. Stewart, Liapunov stability and adding machines, Ergodic Theory Dynam. Systems 15 (1995), 271-290.

[8] W. T. Ingram, Inverse limits on [0,1] using tent maps and certain other piecewise linear bonding maps, in: Continua With the Houston Problem Book (Cincinnati, OH, 1994), H. Cook et al. (eds.), Lecture Notes in Pure Appl. Math. 170, Dekker, 1995, 253-258.

[9] L. B. Jones, Adding machines and endpoints, Topology Appl. 156 (2009), 2899-2905.

[10] L. Kailhofer, A classification of inverse limit spaces of tent maps with periodic critical points, Fund. Math. 177 (2003), 95-120.

[11] W. de Melo and S. van Strien, One-Dimensional Dynamics, Springer, 1993.

[12] B. Raines and S. Stimac, A classification of inverse limit spaces of tent maps with non-recurrent critical point, Algebr. Geom. Topol. 9 (2009), 1049-1088.

[13] S. Štimac, A classification of inverse limit spaces of tent maps with finite critical orbit, Topology Appl. 154 (2007), 2265-2281.

Lori Alvin, Karen Brucks

Department of Mathematical Sciences

University of Wisconsin at Milwaukee

Milwaukee, WI 53201-0413, U.S.A.

E-mail: lmgolner@uwm.edu

kmbrucks@uwm.edu

Received 4 March 2010;

in revised form 13 May 2010 